\title{
Learning in combined-feature search: Specificity to orientation
}

\author{
GIANLUCA CAMPANA and CLARA CASCO \\ Università di Padova, Padova, Italy
}

\begin{abstract}
We investigated the dynamics and specificity of learning in the search for a target defined by combined features belonging to the same dimension (a rotated L) among homogeneous or heterogeneous distractors (differently rotated Ls). We found that learning makes searching faster although the search strategy does not change, remaining parallel with homogeneous distractors and serial with heterogeneous distractors. Learning was found to be specific for combined-feature orientation, although simple features did not change in the transfer stimulus: Transfer was partial when either the target or the distractors were rotated (so that their global orientation became the same) and totally absent when target and distractors were swapped. These results, which apply to searches among both homogeneous and heterogeneous distractors, rule out the possibility that learning is specific for orientation of just simple features. Instead, the results suggest that specificity of learning with combined features reflects the activation of junction detectors responding to a combined feature of a particular orientation.
\end{abstract}

Experience-dependentperceptual improvement (perceptual learning) has been reported in the context of a range of perceptual tasks, such as detection and discrimination of visual gratings (De Valois, 1977; Fiorentini \& Berardi, 1980, 1981; Mayer, 1983), motion direction discrimination (Ball \& Sekuler, 1982, 1987; Ball, Sekuler, \& Machamer, 1983), stimulus orientation judgments (Fahle, 1997; Shiu \& Pashler, 1992; Vogels \& Orban, 1985), and hyperacuity tasks (Beard, Levi, \& Reich, 1995; Bennett \& Westheimer, 1991; Fahle, 1997; Fahle \& Edelman, 1993; Kumar \& Glaser, 1993; McKee \& Westheimer, 1978; Saarinen \& Levi, 1995).

Perceptual learning has recently been found using search tasks involving the detection of an odd element embedded in a background of distractors (Ahissar \& Hochstein, 1996; Casco, Campana, \& Gidiuli, 2001; Ellison \& Walsh, 1998; Karni \& Sagi, 1991, 1993; Sireteanu \& Rettenbach, 1995). When the difference between the odd target and distractors is great enough in some basic dimension (e.g., orientation or size), detection is effortless and search time is fast and independent of the number of distractors displayed (Treisman \& Gelade, 1980). It has been suggested that effortless localization of the odd element is based on local computations allowed by parallel, short-range local inhibitory interactions which reduce the visual system's response to uniform textural fields and enhance textural borders (Casco \& Campana, 1999; Malik \& Perona, 1990; Nothdurft, 1992, 1997; Rubenstein \& Sagi, 1990; Sagi \& Julesz, 1987). The activation of these local mechanisms could mediate effortless target detection on the basis of dis-

We thank three anonymous referees for useful comments on an earlier version of this article. Correspondence should be addressed to C. Casco, Dipartimento di Psicologia Generale, Università degli Studi di Padova, Via Venezia 8, 35131 Padova, Italy (e-mail: clara.casco@ unipd.it). continuities in the feature gradient (Sagi \& Julesz, 1984). Learning in effortless visual search is enduring, since virtually the entire improvement is retained months later. Although contradictory results exist (Sireteanu \& Rettenbach, 1995), it has generally been found that improvement is specific for the basic dimension of the stimulus, since it does not transfer to other values of the same dimension. This specificity has been accounted for in terms of the tuning properties of neurons in the striate (Karni \& Sagi, 1991; 1993; Saarinen \& Levi, 1995) and extrastriate (Ahissar \& Hochstein, 1996) visual cortex. Indeed, if learning results from the tuning of neurons activated at an early level of cortical processing, then it will be specific for the attributes to which these neurons respond. At an early level of central visual processing, these attributes will be orientation, ocular dominance (at least at the level of cortical cells in Layer 4 of V1), spatial frequency, length, and position (at least for simple cells). Support for the high specificity of perceptual learning in effortless visual search of orientation gradient was found by Ahissar and Hochstein. They investigated whether learning is specific for position, size, and orientation of textural elements. Since their results showed strong selectivity for these dimensions, they inferred that learning with such stimuli occurred within an early processing stage in the striate or extrastriate visual areas containing neurons responding to these dimensions.

In the present article, we address the issues of dynamics and specificity of learning in search displays in which the target and distractors are defined by a conjunction of features belonging to the same neural map (i.e., orientation). Although, with a variable target and heterogeneous distractors, this type of search has been found more difficult than between-features conjunction search (Wolfe et al., 1990), search functions with these stimuli are almost flat when distractors are homogeneous and regularly 
arranged (Humphreys, Quinlan, \& Riddoch, 1989), suggesting the involvement of a parallel search strategy. It has been demonstrated that a preattentive mechanism based on short-range inhibitory interactions leading to the detection of the orientation gradient cannot account for parallel searches with these stimuli. Nor can parallel guidance of attention by simple features facilitate search in an array in which simple features are exactly the same in target and distractors elements (Wolfe et al., 1990). However, a parallel search strategy may be used if there is grouping of aligned, regular distractors based on long-range excitatory interactions and via distributed attention (Casco \& Campana, 1999).

Given these results, an important question is whether specificity of learning with such stimuli may be considered in terms of tuning properties of the same mechanisms that respond to the basic dimensions involved in a simplefeature search. This is unlikely, since at high spatial frequencies the target and background elements have the same variability profile across the orientation spectrum. An alternative possibility is that learning is specific for combined features. In this case, specificity may be accounted for in two different ways. One possibility is that learning induces the activation of larger filters tuned to the global orientation of combined features (target, background, or both), since the filter output presents an energy gradient at the target location when the global orientation of the target differs from that of the distractors (Rubenstein \& Sagi, 1990). Alternatively, specificity of learning may be accounted for if learning induces the selection of combined features as a single object (Humphreys \& Muller, 1993; Humphreys et al., 1989), and this may explain why learning does not transfer to a stimulus in which these objects are different. Both these possibilities are physiologically plausible. Indeed, there is evidence that in striate cortex of both cats (Tootel, Silverman, \& De Valois, 1991) and macaque monkeys (Horton \& Hubel, 1981) spatial frequency and orientation are systematically related. As a consequence of perceptual learning, the response of neurons tuned to a particular combination of these two dimensions that makes them sensitive to a single feature of a particular orientation and spatial frequency could be reduced, whereas that of other neurons tuned to a spatial frequency and orientation appropriate for responding optimally to the global orientation of the target combined feature could be enhanced. On the other hand, perceptual learning could increase the neural response to patterns displaying orientation contrast (Kastner, Nothdurft, \& Pigarev, 1997). Indeed, although visually driven cells in the cerebral cortex undoubtedly show selectivity for a certain value of a given dimension, cells being activated by joint sets of properties have also been found (Tanaka, Saito, Fukada, \& Moriya, 1991). In this case, learning would make combined features more salient because it increases the response to a feature template and makes the conjunction target like a single pop-out feature in the visual search task. This development of feature template could involve improvement and/or change of functions carried out by other areas, such as the temporal cortex (Chelazzi, Miller, Duncan, \& Desimone, 1993) and the parietal cortex (Walsh, Ashbridge, \& Cowey, 1998).

To address the issue of dynamics and specificity of learning with combined-feature stimuli, in the present study we first investigated whether learning for combined features is specific for orientation in the same way as learning for simple features is. Second, we aimed to establish whether specificity results from confined receptive field selectivity of the units activated at an early level of processing or from mechanisms responding to more global aspects of the stimulus, perhaps in higher visual areas. To do this, we analyzed the stimulus specificity of learning, assuming that if learning was specific it would have resulted from an improvement in the response of stimulus analyzers which are activated by the learning stimulus in the cortical areas responsible for task performance. Transfer was investigated using stimuli in which the orientation of either simple or combined features changed. Four conditions of transfer were used. In the first transfer condition, the stimulus was the same as the learning stimulus but was rotated by $45^{\circ}$. Transfer of learning to this new stimulus would indicate that the learning mechanism is not specific for either simple-feature or combined-feature orientation. In the second transfer condition, the target was exchanged with the distractors. Since in the transfer stimulus single features do not change, transfer of learning here would indicate that learning is not specific for combined-feature orientation. In the third and fourth transfer conditions, either the target or the distractors were rotated to make the global (i.e., low-pass filtered) orientation of these two combined features the same. If learning increased response of detectors tuned to combined-feature global orientation, it should not transfer to the new stimuli. We found that learning in visual search for combined features is specific for the orientation of the combined features, not just for the simple ones. Indeed, learning does not transfer when the target and background elements are swapped. However, there is substantial transfer when either target or distractors are changed although simple features do not vary, suggesting that learning allows the formation or consolidation of combinedfeature templates, rather than improving detection of global orientation.

\section{METHOD}

\section{Stimuli}

The stimuli are shown in Figures 1 and 2. They consisted of arrays of white combined features $\left(142.4 \mathrm{~cd} / \mathrm{m}^{2}\right)$ presented on a dark background $\left(0.2 \mathrm{~cd} / \mathrm{m}^{2}\right)$. The homogeneous combined feature display consisted of a $180^{\circ}$-rotated L target among $90^{\circ}$-counterclockwiserotated Ls (Figure 1A) or of a $90^{\circ}$-counterclockwise-rotated L target among $180^{\circ}$-rotated Ls (Figure 1B). The heterogeneous combined feature display consisted of a $90^{\circ}$-clockwise-rotated $\mathrm{L}$ target among upright $\mathrm{Ls}, 90^{\circ}$-counterclockwise-rotated $\mathrm{Ls}$, and $180^{\circ}$-rotated Ls (Figure 2A). In $50 \%$ of the trials, the target to be detected was present, and in the remaining trials it was absent. The target (if present) and background elements (distractors) were positionally aligned, and their respective positions were randomly chosen from among the possible array positions. The height and width of feature ele- 
A

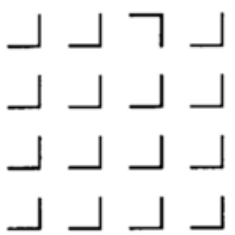

B

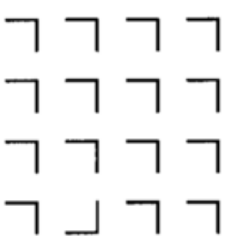

Standard 1 (learning) Standard 2 (learning)

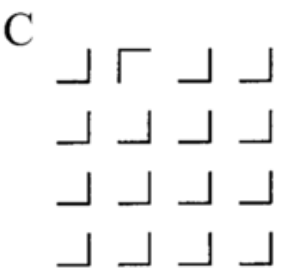

T-rot

$\mathrm{E}$

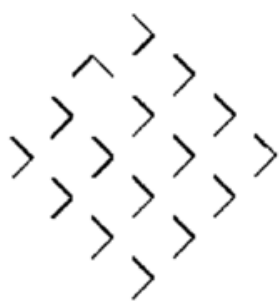

Rot 45
D

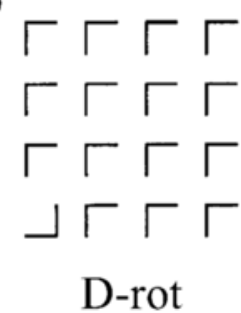

F

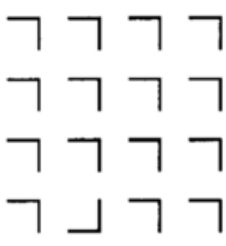

T/D swap
Figure 1. The homogeneous combined-feature displays (examples with set size 16 and target present) used in the learning session (Standard 1 and Standard 2) consisted of either (A) a $180^{\circ}$ rotated $\mathrm{L}$ target in a background of $90^{\circ}$-counterclockwise-rotated Ls or (B) a $90^{\circ}$-counterclockwise-rotated $L$ target in a background of $180^{\circ}$-rotated Ls. T-rot and D-rot are examples of transfer stimuli in which either the target (T-rot, with respect to Standard 1 ) or the distractors (D-rot, with respect to Standard 2) were rotated by $90^{\circ}$. Other stimuli used in the transfer condition (with respect to Standard 1) were Rot 45 (rotation of the entire configuration by $4^{\circ}$ ) and T/D swap (target and distractors swapped with respect to the trained stimulus).

ments in both stimuli were each $30^{\prime}$ of visual angle. Element separation (spacing) was equal to $30^{\prime}$ for all conditions. The three display sizes were of 4,16 , and 36 elements. The display area depended on set size and varied from $1^{\circ} 30^{\prime}$ to $5^{\circ} 30^{\prime}$.

\section{Subjects}

The subjects were 44 undergraduate students, divided into six groups. Four of the groups performed homogeneous distractor search tasks, and two performed heterogeneous distractor search tasks. All of the subjects had normal or corrected-to-normal vision, were not aware of the purpose of the experiment, and had not participated in visual search experiments before.

\section{Apparatus and Procedure}

An IBM PC with a 15 -in. color monitor, $70 \mathrm{~Hz}$ vertical refresh, $800 \times 600$ pixel resolution, and a square pixel of $2.5^{\prime} \times 2.5^{\prime}$ (at $57 \mathrm{~cm}$ viewing distance) was used to generate and present the stimuli and to record the responses.
The temporal sequence of each trial was as follows: The trial started with a fixation mark (a cross with lines $24^{\prime}$ long and with an intensity of $142.4 \mathrm{~cd} / \mathrm{m}^{2}$ ). It was displayed for $1,200 \mathrm{msec}$ and was followed by the stimulus, which remained visible until the subject responded. The subject's response was followed first by feedback (i.e., a display of the search time for correct responses and, for incorrect responses, the "error" signal presented for $1,500 \mathrm{msec}$ together with a $400-\mathrm{Hz}$ tone) and then by a 1,000-msec dark field, after which the fixation point reappeared.

The learning session, performed by 27 of the 44 subjects, consisted of 16 blocks in the homogeneous condition (1,920 trials) and 17 blocks in the heterogeneous condition (1,530 trials) performed on consecutive days. On the day following the last learning block, the transfer session was presented through counterbalancing of the transfer blocks across subjects. Each subject performed either the homogeneous $(n=19)$ or the heterogeneous $(n=8)$ learning and transfer sessions. The control session, viewed by 17 nontrained subjects out of the 44 , consisted of a single block for each of the conditions presented (homogeneous: four conditions; heterogeneous: three conditions). Each subject performed either the homogeneous $(n=10)$ or the heterogeneous $(n=7)$ control session. Each block of randomly presented trials consisted of 20 (homogeneous) or 15 (heterogeneous) repetitions of two stimulus configurations (target present or target absent) and three distractor display sizes $(4,16$, and 36 elements). We had to use fewer repetitions in each heterogeneous block to equate total block duration, since RTs were much longer than in the homogeneous blocks. Six groups of subjects (learning with homogeneous distractors: $n \mathrm{~s}=5,6$, and 8 ; control with homogeneous distractors: $n=10$; learning with heterogeneous distractors: $n=8$; control with heterogeneous distractors: $n=7$ ) performed the search task with different combinations of stimuli, as is described in Table 1. All the subjects who performed the learning session were eventually tested with different stimuli (including display rotated by $45^{\circ}$ [Rot 45], target or distractors rotated [T-rot and D-rot, respectively], and target swapped with distractors [T/D swap]) in a transfer session. The control groups were tested with the standard and transfer stimuli without previous practice on any stimulus. Special care had been taken to ensure that performance in one transfer condition was not affected by performance in another transfer condition in which a similar stimulus configuration was used. This justifies the chosen procedure of running separate groups for each transfer condition (except the Rot 45 condition, which was performed by all the subjects). Sample size was relatively small for the T-rot and D-rot conditions, since in these two conditions the same combined feature was changed (which varied with respect to the related learning stimulus). Note that we had to use two different learning stimuli for these two groups in order to avoid, in either transfer condition, the use of an upright $\mathrm{L}$ as a target or distractor, which could have affected performance owing to familiarity effects.

The subjects' task was to indicate whether the target was present or absent by pressing one of two keys (F8 for present and F5 for absent) on a computer keyboard. The use of the dominant hand for either key was counterbalanced across subjects. Search time for correct responses only was measured independently for target-present and target-absent trials.

\section{Data Analysis: Time Course of Learning}

The time course of learning was assessed by establishing how mean search time in the standard stimuli changed during the learning session. This was done by using an analysis of variance (ANOVA) with stimulus type (standard stimulus of Figure 1A or 1B) as the between-subjects factor (in the homogeneous condition only) and block number ( 16 blocks in the homogeneous displays and 17 in the heterogeneous displays) and response type (target present or target absent) as within-subjects factors. The slope in each block was also calculated to obtain information about whether learning changed the search strategy. Since it is physiologically implausible that serial processing can be realized at a search rate of less than 10 msec (Crick, 
A

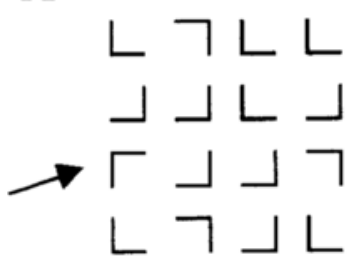

Standard (learning)
B

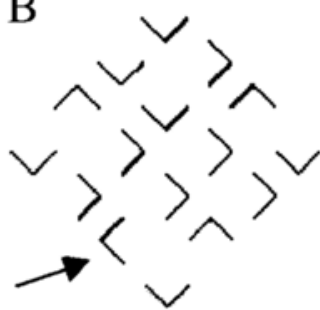

Rot 45
C

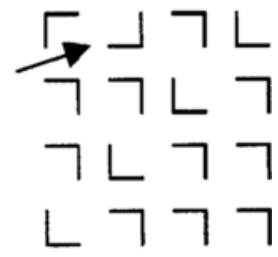

T/D swap

Figure 2. The heterogeneous combined-feature displays (examples with set size 16 and target present) used in the learning session (A) consisted of a $90^{\circ}$-clockwise-rotated $L$ target in a background of upright $\mathrm{Ls}, 9^{\circ}$-counterclockwise-rotated $\mathrm{Ls}$, and $180^{\circ}$-rotated $\mathrm{Ls}$. The transfer stimuli were (B) Rot 45 (rotation of the entire configuration by $45^{\circ}$ ) and (C) T/D swap (the target was swapped with one of the distractors). In each configuration, the target is that indicated by an arrow (not shown in the stimuli presented to the subjects).

1984), slopes larger than $10 \mathrm{msec}$ were interpreted as indicative of exhaustive (if the target-present/ target-absent ratio was close to 1) or self-terminating (if it was close to .5) serial processing. The errors were also analyzed, since search time in conditions with significantly different error rates cannot be compared.

Specificity of learning was investigated using search time as a dependent variable in the following conditions (block types): standard stimulus for control group (standard-before learning), standard in the last block of the learning (standard last), transfer stimulus in the control group (transfer-before learning), and transfer stimulus after learning (transfer-after learning). These search time values allowed us to calculate, for different conditions, the degree of transfer $(T)$ defined as the ratio of the search time difference in the transfer conditions before (transfer before) and after (transfer after) learning and the search time difference in the standard condition before (standard before) and after (standard last) learning, as is shown in Equation 1.

$$
\begin{aligned}
T= & (\text { transfer-before }- \text { transfer-after }) / \\
& \text { (standard before }- \text { standard last })
\end{aligned}
$$

$T$ values range from 0 to 1 . A $T$ equal to 0 is indicative of no transfer, whereas a $T$ equal to 1 denotes a total transfer to the new stimulus.

Note that the calculation of $T$ was necessary because a simple difference between standard last and transfer after (usually taken as an index of degree of transfer in the literature on perceptual learning) could result from factors other than learning — that is, from a differ- ence in discriminability between standard (standard-before) and transfer (transfer-after) stimuli.

To establish whether the value of $T$ for each of the four transfer stimuli differed significantly from that obtained in a hypothetical condition in which the transfer stimulus was the same as the learning stimulus (and transfer was therefore total), we first calculated the $T$ in this hypothetical condition. To do this, we used the mean search time obtained in standard (last-1) as a standard-last value and that obtained in standard last as a transfer-after value. The difference between the values of $T$ was checked for significance using a chisquared technique, ${ }^{1}$ comparing the $T$ value obtained in each condition and the $T$ value obtained in the previously described hypothetical condition (i.e., different stimuli vs. same stimulus) with an ideal condition in which the transfer was total and the $T$ value was equal to $1 .^{2}$ In other words, we assessed whether our $T$ values were significantly different from 1 . A lack of significance was assumed to indicate that learning transferred to the new stimulus.

\section{RESULTS}

\section{Time Course of Learning}

The time course of learning is shown in Figure 3 by a plot of mean search time (i.e., the search time averaged across display size) in homogeneous (panel A, $n=19$ ) and heterogeneous ( panel $\mathrm{B}, n=8$ ) conditions as a func-

Table 1

\begin{tabular}{|c|c|c|c|c|c|c|c|c|}
\hline \multirow[b]{2}{*}{ Distractors } & \multirow[b]{2}{*}{ Group } & \multicolumn{3}{|c|}{ Standard Homogeneous Stimuli } & \multicolumn{4}{|c|}{ Transfer Stimuli } \\
\hline & & $\begin{array}{c}\text { Training } \\
\text { (Figure 1A) }\end{array}$ & $\begin{array}{l}\text { Training } \\
\text { (Figure 1B) }\end{array}$ & $\begin{array}{c}\text { Training } \\
\text { (Figure 2A) }\end{array}$ & $\begin{array}{c}\text { T-rot } \\
\text { (Figure 1C) }\end{array}$ & $\begin{array}{c}\text { D-rot } \\
\text { (Figure 1D) }\end{array}$ & $\begin{array}{c}\text { Rot } 45 \\
\text { (Figure 1E) }\end{array}$ & $\begin{array}{c}\text { T/D swap } \\
\text { (Figure 1F) }\end{array}$ \\
\hline \multicolumn{9}{|l|}{ Homogeneous } \\
\hline \multirow[t]{3}{*}{ Learning session } & $1(n=5)$ & & $*$ & & & $*$ & $*$ & \\
\hline & $2(n=6)$ & $*$ & & & $*$ & & $*$ & \\
\hline & $3(n=8)$ & $*$ & & & & & $*$ & $*$ \\
\hline Control session & $4(n=10)$ & $*$ & & & $*$ & $*$ & $*$ & * \\
\hline Heterogeneous & & & & & & & $\begin{array}{c}\text { Rot } 45 \\
\text { (Figure 2B) }\end{array}$ & $\begin{array}{l}\text { T/D swap } \\
\text { (Figure 2C) }\end{array}$ \\
\hline Learning session & $5(n=8)$ & & & $*$ & & & $*$ & $*$ \\
\hline Control session & $6(n=7)$ & & & $*$ & & & $*$ & $*$ \\
\hline
\end{tabular}

Conditions Presented to Each Group of Subjects

Note-For Group 4, performance in the standard homogeneous condition of Figure 1B and the T/D swap condition was assumed to be the same as performance on the standard stimulus of Figure 1A. Performance in D-rot (Figure 1D) was assumed to be the same as that in T-rot. 

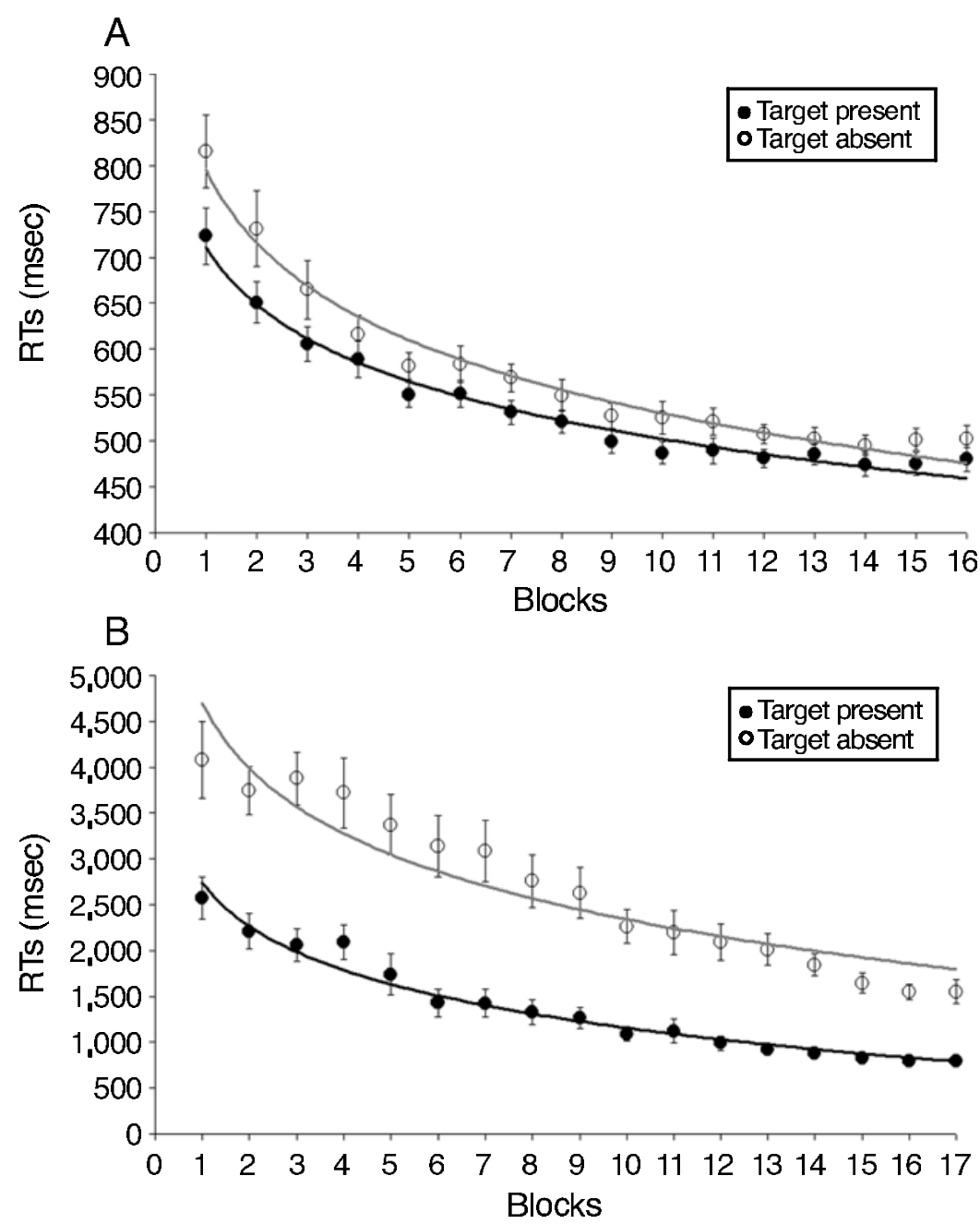

Figure 3. Mean search time (in milliseconds) as a function of block number for target-present (filled symbols) and target-absent (unfilled symbols) responses. Data obtained with the homogeneous display are shown in panel $A$, and those obtained with the heterogeneous display are shown in panel $B$. Error bars indicate intersubject standard errors $(S E S)$. In both conditions, the rate of improvement is captured by a logarithmic decay. The mean search-time improvement equation for the homogeneous display was equal to $y=-90.941 \ln (x)+711.52\left(R^{2}=.98\right)$ for the target-present response and to $y=-115.35 \ln (x)+795.76\left(R^{2}=.98\right)$ for the target-absent responses, whereas that for the heterogeneous display was equal to $y=-689.08 \ln (x)+2,743.5$ $\left(R^{2}=.97\right)$ for the target-present response and $y=-1022.8 \ln (x)+4,695.5\left(R^{2}=.88\right)$ for the target-absent response.

tion of block number, separately for target-present and target-absent responses. Note that we merged data from all the subjects who performed the homogeneous learning session. In the homogeneous condition, search time did not depend on which learning stimulus was used [Standard 1, shown in Figure 1A, or Standard 2, shown in Figure 1B: $F(1,17)=0.3, p>.05]$. The effect of block number was significant in both homogeneous $[F(15,255)=$ $39.9, p<.0001]$ and heterogeneous $[F(16,112)=30.3$, $p<.0001]$ conditions. The effect of response type [homogeneous, $F(1,17)=12.9, p<.01$; heterogeneous,
$F(1,16)=76.8, p<.0001]$ and the interaction between block and response type [homogeneous, $F(15,255)=4.0$, $p<.0001$; heterogeneous, $F(16,112)=9.1, p<.0001]$ were also significant. Simple contrasts show that the differences between the first and last blocks in the learning session were significant for both homogeneous $[F(1,17)=$ $70.1, p<.0001]$ and heterogeneous $[F(1,7)=45.7, p<$ $.0001]$ conditions. This indicates that learning generally reduces search time for all stimuli and conditions. We found a fast initial improvement in the first four blocks, although further practice induced further improvement. 
The rate of improvement is captured by a single logarithmic decay, $y=a-b *(\log x)$, where the parameters are $y$ (mean search time), $a$ (maximum mean search time in the first block), $b$ (learning rate constant), and $x$ (block number). The smooth curves in Figure 3 are drawn by the best fit to the above equation. The learning rate constant is as follows: homogeneous present $\left(91 \mathrm{msec}, R^{2}=\right.$ $.98)$, homogeneous absent ( $115 \mathrm{msec}, R^{2}=.97$ ), heterogeneous present $\left(689 \mathrm{msec}, R^{2}=.96\right)$, and heterogeneous absent $\left(1,023 \mathrm{msec}, R^{2}=.88\right)$.

The data were further analyzed to calculate the slopes of the functions relating search time to element display size, independently for target-present and target-absent trials (Tables 2A and 2B).

With homogeneous distractors (Table 2A), mean slopes are very low for both target-present and target-absent trials (less than $2 \mathrm{msec}$ per item on average). This floor effect excludes the possibility that they can be used as dependent variables to analyze learning dynamics and specificity. With heterogeneous distractors (Table 2B), the slopes generally decrease as block number increases (target present, from 78 to $13 \mathrm{msec}$ per item; target absent, from 141 to $51 \mathrm{msec}$ per item) with the target-present: target-absent ratio shifting from .55 to .25 before and after learning. The target-present:target-absentratio before and after learning shows a similar trend when mean search time is considered (from .89 to .95 with homogeneous displays and from .63 to .52 with heterogeneous displays). This indicates that after learning, searching in homogeneous distractors remains parallel, whereas searching in heterogeneous displays remains serial self-terminating. Consequently, it is unlikely that learning effects reflect a change in the attentional demands in the search task. In agreement with our interpretation, other authors (Lobley \& Walsh, 1998; Treisman, Vieira, \& Hayes, 1992) suggest that learning in conjunction search depends on changes that are specific to the particular stimulus rather than on improvement of cognitive strategies common to all search tasks (Sireteanu \& Rettenbach, 1995).

\section{Specificity of Learning}

Display orientation change. In this transfer condition, the entire configuration was rotated by $45^{\circ}$. This condition was performed in the transfer session by 19 subjects in the homogeneous condition and by 8 subjects in the heterogeneous condition.

In the search with homogeneous distractors, the degree of transfer $T=$ (transfer-before - transfer-after) $/$ (standard before - standard last $)$ was equal to $.20\left(\chi_{1}=33.1, p<\right.$ $.001)$ and $.36\left(\chi_{1}=17.6, p<.001\right)$ for the target-present

Table 2A

Mean Search Times (in Milliseconds), Slopes and Intercepts (in Milliseconds per Element), and Goodness of Fit $\left(R^{2}\right)$ of the Functions Relating Search Time to Set Size for Target-Present and Target-Absent Trials for the Homogeneous Displays, for All Blocks of the Learning Session and for All Blocks of the Transfer and Control Sessions

\begin{tabular}{|c|c|c|c|c|c|c|c|c|}
\hline & \multicolumn{4}{|c|}{ Target Present } & \multicolumn{4}{|c|}{ Target Absent } \\
\hline & $\begin{array}{c}\text { Mean } \\
\text { Search Time }\end{array}$ & Slope & Intercept & $R^{2}$ & $\begin{array}{c}\text { Mean } \\
\text { Search Time }\end{array}$ & Slope & Intercept & $R^{2}$ \\
\hline \multicolumn{9}{|l|}{$\begin{array}{l}\text { Learning blocks } \\
\qquad(n=19)\end{array}$} \\
\hline 1 & 723 & 2.50 & 677 & .87 & 816 & 8.11 & 664 & 1.00 \\
\hline 2 & 651 & 0.49 & 642 & .10 & 731 & 6.14 & 617 & .98 \\
\hline 3 & 606 & 1.59 & 576 & .79 & 665 & 5.46 & 563 & .99 \\
\hline 4 & 589 & 1.38 & 563 & .84 & 617 & 3.85 & 545 & 1.00 \\
\hline 5 & 550 & 1.61 & 520 & .85 & 582 & 2.70 & 531 & 1.00 \\
\hline 6 & 551 & 1.38 & 525 & .56 & 584 & 4.05 & 509 & 1.00 \\
\hline 7 & 531 & 1.00 & 512 & .88 & 569 & 3.13 & 510 & .97 \\
\hline 8 & 521 & 1.17 & 500 & .77 & 550 & 2.75 & 498 & 1.00 \\
\hline 9 & 500 & 1.16 & 478 & .86 & 527 & 3.10 & 470 & .96 \\
\hline 10 & 487 & 0.85 & 471 & .71 & 525 & 3.33 & 463 & .98 \\
\hline 11 & 489 & 1.24 & 466 & .97 & 521 & 2.66 & 471 & .97 \\
\hline 12 & 481 & 0.32 & 475 & .14 & 507 & 2.85 & 454 & .96 \\
\hline 13 & 486 & 1.48 & 458 & .91 & 502 & 1.92 & 466 & .98 \\
\hline 14 & 463 & 0.45 & 454 & .90 & 485 & 0.79 & 470 & .77 \\
\hline 15 & 465 & -0.01 & 465 & .00 & 496 & 0.39 & 489 & .64 \\
\hline 16 & 474 & 0.30 & 468 & .29 & 499 & 0.54 & 489 & .59 \\
\hline \multicolumn{9}{|l|}{ Transfer blocks* } \\
\hline $\operatorname{Rot} 45(n=19)$ & 567 & 3.01 & 510 & 0.99 & 608 & 6.5 & 486 & .99 \\
\hline T-rot $(n=6)$ & 476 & 0.8 & 461 & 0.95 & 497 & 1.1 & 477 & .97 \\
\hline D-rot $(n=5)$ & 477 & 0.5 & 468 & 0.24 & 535 & 0.5 & 524 & .48 \\
\hline T/NT swap $(n=8)$ & 623 & 5.5 & 521 & 0.96 & 666 & 8.7 & 504 & .98 \\
\hline \multicolumn{9}{|c|}{ Control blocks $(n=10) \dagger$} \\
\hline Standard 1 & 583 & 0.4 & 587 & .05 & 604 & 2.7 & 554 & .90 \\
\hline Rot 45 & 589 & 1.3 & 564 & .99 & 646 & 4.5 & 562 & .99 \\
\hline T- or D-rot & 584 & 1.5 & 556 & .89 & 610 & 3.1 & 551 & .97 \\
\hline
\end{tabular}

Note_* Transfer after. †Standard and transfer before. Note that Standard 1 is also used as a control for T/D swap, since the two stimuli are virtually the same. T/NT swap, target-nontarget swap. 
Table 2B

Mean Search Times (in Milliseconds), Slopes and Intercepts (in Milliseconds per Element), and Goodness of Fit $\left(R^{2}\right)$ of the Functions Relating Search Time to Set Size for Target-Present and Target-Absent Trials for the Heterogeneous Displays, for All Blocks of the Learning Session and for All Blocks of the Transfer and Control Sessions

\begin{tabular}{|c|c|c|c|c|c|c|c|c|}
\hline & \multicolumn{4}{|c|}{ Target Present } & \multicolumn{4}{|c|}{ Target Absent } \\
\hline & $\begin{array}{c}\text { Mean } \\
\text { Search Time }\end{array}$ & Slope & Intercept & $R^{2}$ & $\begin{array}{c}\text { Mean } \\
\text { Search Time }\end{array}$ & Slope & Intercept & $R^{2}$ \\
\hline \multicolumn{9}{|c|}{$\begin{array}{l}\text { Learning Blocks } \\
\quad(n=8)\end{array}$} \\
\hline 1 & 2,572 & 77.74 & 1,121 & 1.00 & 4,080 & 141.11 & 1,446 & .98 \\
\hline 2 & 2,209 & 72.80 & 850 & 1.00 & 3,744 & 126.76 & 1,378 & .98 \\
\hline 3 & 2,061 & 53.64 & 1,060 & .86 & 3,878 & 145.77 & 1,157 & .99 \\
\hline 4 & 2,093 & 76.39 & 667 & 1.00 & 3,722 & 137.66 & 1,152 & .97 \\
\hline 5 & 1,742 & 50.46 & 800 & .98 & 3,370 & 127.87 & 984 & .98 \\
\hline 6 & 1,429 & 36.41 & 749 & .96 & 3,137 & 125.54 & 794 & .99 \\
\hline 7 & 1,423 & 43.06 & 619 & 1.00 & 3,081 & 113.66 & 959 & .98 \\
\hline 8 & 1,326 & 33.17 & 707 & .97 & 2,758 & 101.09 & 871 & .99 \\
\hline 9 & 1,268 & 31.49 & 680 & 1.00 & 2,630 & 94.59 & 864 & .97 \\
\hline 10 & 1,092 & 27.14 & 586 & .99 & 2,265 & 80.35 & 765 & .99 \\
\hline 11 & 1,123 & 26.97 & 619 & 1.00 & 2,197 & 67.84 & 930 & .94 \\
\hline 12 & 992 & 19.50 & 628 & .98 & 2,091 & 69.09 & 801 & .99 \\
\hline 13 & 921 & 14.84 & 644 & .99 & 2,013 & 67.05 & 761 & .98 \\
\hline 14 & 881 & 12.42 & 650 & .99 & 1,844 & 54.95 & 818 & .97 \\
\hline 15 & 827 & 14.37 & 559 & 1.00 & 1,647 & 51.57 & 685 & .99 \\
\hline 16 & 792 & 11.31 & 581 & 1.00 & 1,548 & 39.01 & 819 & .95 \\
\hline 17 & 800 & 13.00 & 557 & 1.00 & 1,550 & 51.40 & 590 & 1.00 \\
\hline \multicolumn{9}{|c|}{ Transfer blocks $(n=8)$} \\
\hline Rot 45 & 1,915 & 53.19 & 922 & .97 & 3,849 & 136.89 & 1,294 & .99 \\
\hline T/D swap & 2,349 & 66.86 & 1,101 & .98 & 4,018 & 141.81 & 1,371 & .99 \\
\hline \multicolumn{9}{|c|}{ Control blocks $(n=7)$} \\
\hline Standard 1 & 2,366 & 55 & 1,343 & 1 & 3,816 & 120 & 1,570 & 1 \\
\hline Rot 45 & 1,971 & 41 & 1,206 & 1 & 3,356 & 104 & 1,410 & 1 \\
\hline T/D swap & 2,221 & 64 & 1,027 & 1 & 3,750 & 122 & 1,478 & 1 \\
\hline
\end{tabular}

and target-absent conditions, respectively (Figure 4). In the search with heterogeneous distractors, $T$ was equal to $.02\left(\chi_{1}=66.9, p<.001\right)$ for the target-present condition and $0\left(\chi_{1}=72.1, p<.001\right)$ for target-absent condition. The effect of transfer was significant in all these condi- tions, indicating that transfer to the $45^{\circ}$ rotated displays was either small (in the homogeneous displays) or absent (in the heterogeneous displays). Since the rotation of the display changes both single- and combined-feature orientation, whereas the relative position remained fixed, the

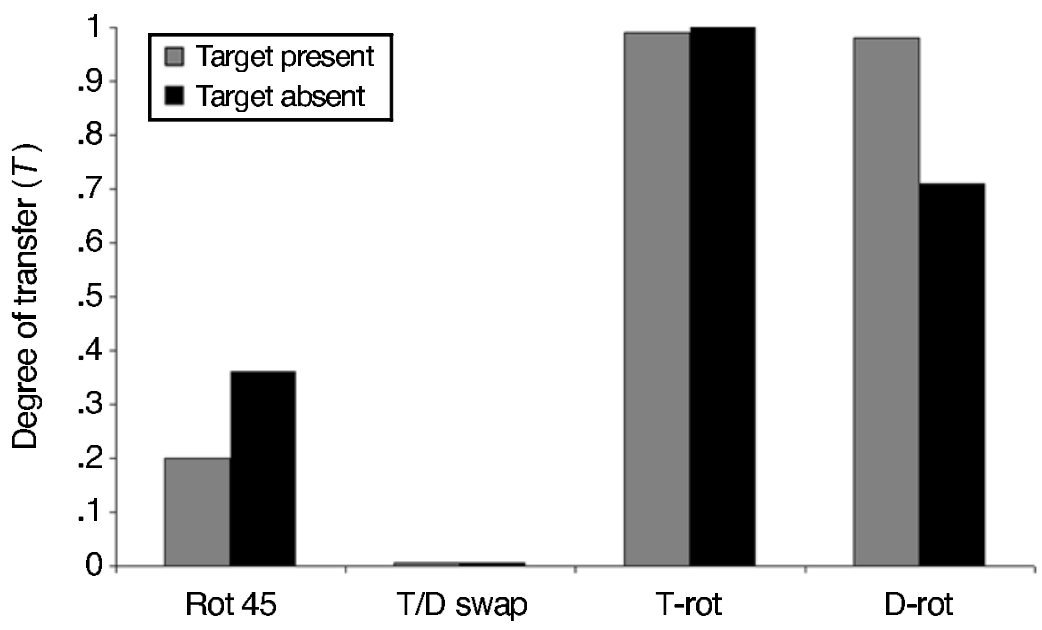

Figure 4. The degree of transfer $(T)$ to different stimuli for homogeneous conditions for target-present and target-absent responses. 
question is whether the learning process increases the orientation selectivity of simple-feature or combined-feature analyzers.

T/D swap. To answer this question, another orientation change was tested in the transfer session with one group of subjects $(n=8)$ in the homogeneous condition and one group $(n=8)$ in the heterogeneous condition (the [T/D] swap).

The degree of transfer $T=$ (transfer-before - transferafter) / (standard before - standard last) is 0 for target present and target absent for both homogeneous (target present: $\chi_{1}=71.5, p<.001$; target absent: $\chi_{1}=72.8, p<$ .001 ) and heterogeneous (target present: $\chi_{1}=72.1, p<$ .001 ; target absent: $\left.\chi_{1}=72.1, p<.001\right)$ displays. The chisquared test was significant, indicating that there is no transfer of learning to a stimulus in which target and distractors are swapped. These results suggest that learning is more specific for combined-feature orientation than for simple-feature orientation, since in this transfer stimulus single-feature orientation does not change.

Target or distractors orientation change. Learning specificity for combined features could be explained in two ways. It may contribute to the formation of an orientationspecific combined-feature representation (i.e., a template), or it could induce activation of a larger filter tuned to the global orientation of combined features (either the target or the distractors), since the filter output presents an energy gradient at target location when the global ori-
A
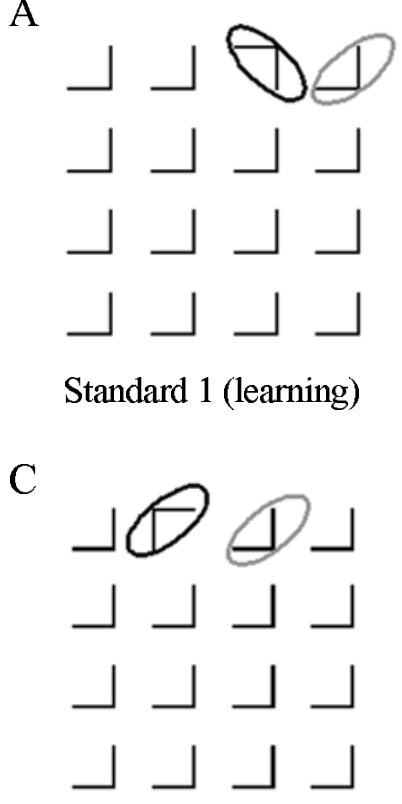

T-rot
Standard 1 (learning)
$\mathrm{B}$

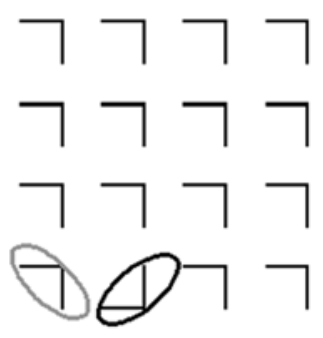

Standard 2 (learning)

$\mathrm{D}$

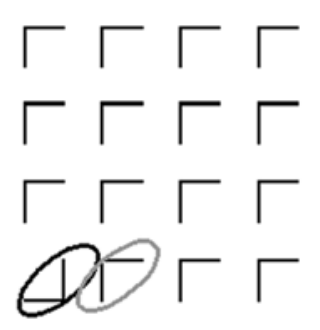

D-rot
Figure 5. Target (in red) and distractors (in green) global orientation for homogeneous stimuli in learning (Standard 1 and Standard 2), T-rot, and D-rot transfer conditions. In T-rot and Drot, there is no global orientation gradient. entation of target and that of the distractors differ (Rubenstein \& Sagi, 1990). To choose between these two possibilities, a transfer condition (for the homogeneous display only) was used with a crucial constraint: Whereas in the learning condition the global orientation of target and distractors was different, such that an orientation gradient was present, in the transfer condition it was kept identical, such that no orientation gradient was present at the level of global orientation, on the basis of which the target location could be detected (Figure 5). If learning increases the response for the global orientation of target and distractors, with this transfer condition it should be greatly hindered.

Two groups of subjects were actually tested for search with two different stimuli having no global orientation gradient: either a D-rot of $90^{\circ}$ or a T-rot of $90^{\circ}$.

Transfer in the D-rot condition was tested in one group $(n=5)$ for search in the homogeneous distractor display. The degree of transfer $T=$ (transfer-before - transfer-after) / (standard before - standard last) is .98 $\left(\chi_{1}=0, p>.05\right)$ for target present and .71 $\left(\chi_{1}=2.3, p>.05\right)$ for target absent (Figure 4$)$. The chi-squared test was not significant, indicating that a large amount of learning transfers to a stimulus in which the distractors are rotated by $90^{\circ}$.

Transfer in the T-rot condition was tested in another group of subjects $(n=6)$ for search with homogeneous distractors. The degree of transfer in the homogeneous display is $.99\left(\chi_{1}=0, p>.05\right)$ for the target-present and $1 \chi_{1}=0$, $p>.05$ ) for the target-absent condition (Figure 4), and the chi-squared test was not significant. Recalling that in the learning stimulus the global orientation of target was different from that of the distractors and in the transfer stimulus it was the same, we expected no transfer if learning increased the responses to the global orientation of target and distractors. The results instead indicate that most of the learning transfers to a stimulus in which the target or the distractors are rotated $90^{\circ}$. This result rules out the filtering explanation and suggests that subjects learn to form a combined-feature representation (template), which is combined-orientation specific.

\section{Error Analysis}

Errors (in all stimulus conditions) are reported independently for each set size in Tables 3A and 3B.

An ANOVA showed that for search with heterogeneous distractors, error rate was larger in the target-present trials [heterogeneous learning: $F(1,7)=16.0, p<.005$; heterogeneous-control: $F(1,6)=19.1, p<.005$ ], whereas in the search with homogeneous distractors, the error rates for target-present and target-absent conditions were similar [homogeneous learning: $F(1,17)=1.2, p>.05$; homogeneous control: $F(1,9)=3.4, p>.05]$. Moreover, the ANOVA showed that only in search with heterogeneous distractors (1) learning reduced error rates $[F(16,112)=$ $9.9, p<.0001]$ and $(2)$ errors increased significantly as set size increased $[F(1,14)=7.8, p<.005]$, mainly in the first sessions and only for target-present trials $[F(32,224)=$ $2.3, p=.0001]$. These results indicate that the effect of 
Table 3A

Percentage of Errors, Averaged Across Subjects, for Each Set Size Level and for Target-Present and Target-Absent Trials for the Homogeneous Displays, for All Blocks of the Learning Session and for All Blocks of the Transfer and Control Sessions

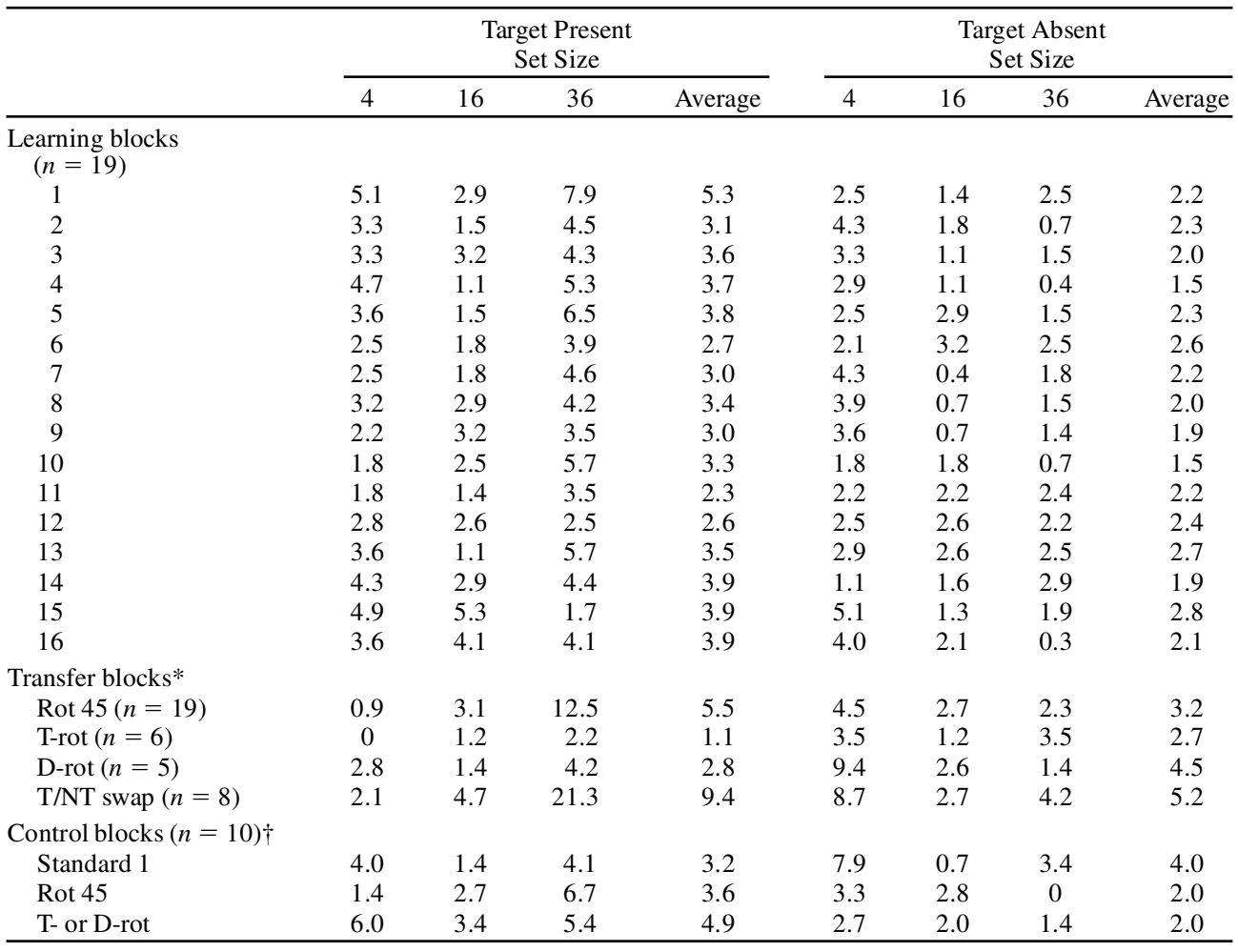

Note-*Transfer after. ${ }^{\dagger}$ Standard and transfer before. Note that Standard 1 is also used as a control for T/D swap, since the two stimuli are virtually the same. T/NT swap, target-nontarget swap.

learning resulting from search time is, in these conditions, underestimated.

The difference in error rate before and after learning is generally smaller for the transfer stimuli, indicating that transfer has been overestimated. This is always true except in the case of search among homogeneous distractors, in the T-rot and D-rot conditions when the target is present, where this difference is smaller for the transfer stimuli, indicating that the degree of transfer found with the $T$ values might have been underestimated. Overall, the results obtained by computing $T$ values and error rates coincide.

\section{CONCLUSION}

Our results show that the degree of transfer is very different between conditions. This allows the first important question concerning learning with combined features to be answered: that of whether learning is specific for the stimulus (Ahissar \& Hockstein, 1996; Carrasco, Ponte, Rechea, \& Sampedro, 1998; Lobley \& Walsh, 1998) or constrained to a change in high-level cognitive strategies (Sireteanu \& Rettenbach, 1995). In both homogeneous and heterogeneous displays, the degree of transfer is small or absent when target and/or distractors are swapped or ro- tated by $45^{\circ}$, and large (for the homogeneous condition only) when either the target or the distractors are changed. These results are in agreement with the view of Lobley and Walsh that perceptual learning of visual conjunction search is constrained mainly by the stimulus parameters rather than by change in cognitive strategies.

The results also show that exchanging the target with distractors hinders learning even if simple features remain unchanged. This rules out the possibility that, with combinedfeature stimuli, learning improves detection of simple features (Ahissar \& Hochstein, 1996).

Our results also preclude the possibility that learning induces the activation of a larger filter tuned to the global orientation of combined features (Malik \& Perona, 1990; Rubenstein \& Sagi, 1990). Indeed, such a mechanism would respond when the global orientations of target and distractors differ. This is the case in our learning condition, but not in the two transfer conditions, in which either the target or the distractors change. In these two conditions, the global orientation is the same and target detection cannot be accounted for on the basis of this filtering model. Moreover, our results do not support the obvious suggestion that, since the search for combined features is mediated by distractor grouping (Duncan \& Humphreys, 
Table 3B

Percentage of Errors, Averaged Across Subjects, for Each Set Size Level and for

Target-Present and Target-Absent Trials for the Heterogeneous Displays, for All Blocks of the Learning Session and for All Blocks of the Transfer and Control Sessions

\begin{tabular}{|c|c|c|c|c|c|c|c|c|}
\hline & \multicolumn{4}{|c|}{$\begin{array}{l}\text { Target Present } \\
\text { Set Size }\end{array}$} & \multicolumn{4}{|c|}{$\begin{array}{c}\text { Target Absent } \\
\text { Set Size }\end{array}$} \\
\hline & 4 & 16 & 36 & Average & 4 & 16 & 36 & Average \\
\hline \multicolumn{9}{|c|}{$\begin{array}{l}\text { Learning Blocks } \\
\quad(n=8)\end{array}$} \\
\hline 1 & 5 & 25 & 22.5 & 17.5 & 0 & 0 & 1.3 & 0.4 \\
\hline 2 & 2.5 & 12.5 & 22.5 & 12.5 & 0 & 0 & 0 & 0 \\
\hline 3 & 2.5 & 7.5 & 11.3 & 7.1 & 0 & 0 & 0 & 0 \\
\hline 4 & 0 & 6.3 & 16.3 & 7.5 & 2.5 & 1.3 & 1.3 & 1.7 \\
\hline 5 & 1.3 & 2.5 & 5 & 2.9 & 0 & 2.5 & 0 & 0.8 \\
\hline 6 & 2.5 & 7.5 & 3.8 & 4.6 & 0 & 0 & 0 & 0 \\
\hline 7 & 1.3 & 1.3 & 3.8 & 2.1 & 1.3 & 0 & 0 & 0.4 \\
\hline 8 & 2.5 & 6.3 & 6.3 & 5 & 0 & 0 & 0 & 0 \\
\hline 9 & 1.3 & 1.3 & 2.5 & 1.7 & 1.3 & 0 & 0 & 0.4 \\
\hline 10 & 2.5 & 2.5 & 0 & 1.7 & 0 & 0 & 1.3 & 0.4 \\
\hline 11 & 2.5 & 0 & 0 & 0.8 & 0 & 0 & 1.3 & 0.4 \\
\hline 12 & 1.3 & 3.8 & 2.5 & 2.5 & 0 & 1.3 & 0 & 0.4 \\
\hline 13 & 3.8 & 1.3 & 2.5 & 2.5 & 1.3 & 2.5 & 0 & 1.3 \\
\hline 14 & 0 & 0 & 3.8 & 1.3 & 0 & 0 & 0 & 0 \\
\hline 15 & 1.3 & 2.5 & 0 & 1.3 & 1.3 & 0 & 0 & 0.4 \\
\hline 16 & 0 & 0 & 1.3 & 0.4 & 0 & 0 & 0 & 0 \\
\hline 17 & 2.5 & 2.5 & 1.3 & 2.1 & 0 & 1.3 & 0 & 0.4 \\
\hline \multicolumn{9}{|c|}{ Transfer blocks $(n=8)$} \\
\hline $\operatorname{Rot} 45^{\circ}$ & 6.3 & 10.0 & 21.3 & 12.5 & 1.3 & 1.3 & 1.3 & 1.3 \\
\hline T/D swap & 7.5 & 13.8 & 36.3 & 19.2 & 1.3 & 0.0 & 1.3 & 0.8 \\
\hline \multicolumn{9}{|c|}{ Control blocks $(n=7)$} \\
\hline Standard & 8.6 & 12.9 & 35.7 & 19.0 & 0 & 4.3 & 20.0 & 8.1 \\
\hline $\operatorname{Rot} 45^{\circ}$ & 17.1 & 25.7 & 30.0 & 24.3 & 1.4 & 5.7 & 11.4 & 6.2 \\
\hline T/D swap & 11.4 & 31.4 & 37.1 & 26.7 & 0 & 1.4 & 15.7 & 5.7 \\
\hline
\end{tabular}

1989), learning makes the grouping strategy more efficient (Casco et al., 2001). If this were the case, we would expect a lack of transfer to occur in heterogeneous but not in homogeneous displays, which is contrary to what we found.

To explain our results, which show that transfer is almost entirely absent in a stimulus in which target and distractors are swapped (where simple features remain the same) and is partial when either target or distractors are rotated, we suggest that the learning process makes combined features more salient. Our results suggest that learning activates junction detectors responding to combined features of a particular orientation and makes target and background element representation more salient. Indeed, regardless of search strategy (through parallel search strategy with homogeneous distractors or through serial scanning with heterogeneous distractors), transfer is possible only if the combined feature of either element of the learning stimulus (target or distractor) remains the same in the transfer stimulus. The formation of an orientation-specific combined-feature representation (template) could depend on a short-range excitatory mechanism allowing the simple combination of form features (such as rotated Ls) to be coded in specific junction detectors. Physiological support for this hypothesis has been provided. For example, Kastner et al. (1997) showed that neurons in the striate cortex respond well to patterns displaying feature contrast, and Tanaka et al. (1991) found cells in the visual cortex that are activated by joint sets of properties.
The response of the mechanism giving optimal response when stimulated by joint sets of features (e.g., simple orientations) could be enhanced by learning in order to increase the saliency of these stimuli. Chelazzi et al. (1993) and Walsh et al. (1998) suggest that this may occur as a result of an improvement or change of functions of visual areas at a level above V1. Indeed, we found, in experiments not reported here, that monocular learning in search with combined-feature stimuli does transfer to the nonstimulated eye, and therefore this possibility cannot be discarded.

This account of perceptual improvement is in agreement with the suggestions (Braun, 1999) that the strength of spatial interactions does not remain fixed, but when a visual pattern is observed, it selectively adapts to local stimulus configurations. If governed by the right rules, this plasticity can ensure that the strength of spatial interactions reflects global stimulus properties. In attempting to explain this dynamic mechanism of learning, one could refer to the basic assumption put forward by Hebb (1949), according to which the effectiveness of a synapse is increased every time it is able to activate the postsynaptic neurons. If this assumption holds for short-range spatial interactions (i.e., interactions between the bars forming a combined feature), it is possible that the simultaneous and repeated presentation of a junction configuration (L) made up of features presenting orientation contrast increases the effectiveness of the synapses between the neurons responding to the two features. 


\section{REFERENCES}

AhISSAR, M., \& HochsteIn, S. (1996). Learning pop-out detection: Specificities to stimulus characteristics. Vision Research, 36, 3487-3500.

BALl, K., \& SEKUler, R. (1982). A specific and enduring improvement in visual motion discrimination. Science, 218, 697-698.

BALl, K., \& SeKuler, R. (1987). Direction-specific improvement in motion discrimination. Vision Research, 27, 953-956.

Ball, K., Sekuler, R., \& Machamer, J. (1983). Detection and identification of moving targets. Vision Research, 23, 229-238.

Beard, B. L., Levi, D. M., \& Reich, L. N. (1995). Perceptual learning in parafoveal vision. Vision Research, 35, 1679-1690.

Bennett, R. G., \& Westheimer, G. (1991). The effect of training on visual alignment discrimination and grating resolution. Perception \& Psychophysics, 49, 541-546.

BraUn, J. (1999). On the detection of salient contours. Spatial Vision, 12, 211-225.

Carrasco, M., Ponte, D., Rechea, C., \& Sampedro, M. J. (1998). "Transient structures": The effects of practice and distractor grouping on within-dimension conjunction searches. Perception \& Psychophysics, 60, 1243-1258

Casco, C., \& Campana, G. (1999). Spatial interactions in simple and combined-feature visual search. Spatial Vision, 12, 467-483.

Casco, C., Campana, G., \& Gidiuli, O. (2001). Stimulus specific dynamics of learning in conjunction search tasks. Visual Cognition, 8 , 145-162.

Chelazzi, L., Miller, E. K., Duncan, J., \& Desimone, R. (1993). A neural basis for visual search in inferior temporal cortex. Nature, $\mathbf{3 6 3}$, 345-347.

CrICK, F. (1984). Function of the thalamic reticular complex: The search for light hypothesis. Proceedings of the National Academy of Sciences, 81, 4586-4590.

De VALoIS, K. K. (1977). Spatial frequency adaptation can enhance contrast sensitivity. Vision Research, 17, 1057-1065.

Duncan, J., \& Humphreys, G. W. (1989). Visual search and stimulus similarity. Psychological Review, 96, 433-458.

Ellison, A., \& WALSH, V. (1998). Perceptual learning in visual search: Some evidence of specificities. Vision Research, 38, 333-345.

FAHLE, M. (1997). Specificity of learning curvature, orientation, and vernier discriminations. Vision Research, 37, 1885-1895.

FAHLE, M., \& EDELMAN, S. (1993). Long-term learning in vernier acuity: Effects of stimulus orientation, range and of feedback. Vision Research, 33, 397-412.

Fiorentini, A., \& Berardi, N. (1980). Perceptual learning specific for orientation and spatial frequency. Nature, 287, 43-44.

Fiorentini, A., \& Berardi, N. (1981). Learning in grating waveform discrimination: Specificity for orientation and spatial frequency. $\mathrm{Vi}$ sion Research, 21, 1149-1158.

Heвв, D. O. (1949). The organization of behavior: A neuropsychological theory. New York: Wiley.

Horton, J. C., \& Hubel, D. H. (1981). Regular patchy distribution of cytochrome-oxidase staining in primary visual cortex of macaque monkey. Nature, 292, 762-764.

Humphreys, G. W., \& Muller, H. J. (1993). SEarch via Recursive Rejection (SERR): A connectionist model of visual search. Cognitive Psychology, 25, 43-110.

Humphreys, G. W., Quinlan, P. T., \& RidDOch, M. J. (1989). Grouping processes in visual search: Effects with single- and combined-feature targets. Journal of Experimental Psychology: General, 118, 258-279.

KARNI, A., \& SAGI, D. (1991). Where practice makes perfect in texture discrimination: Evidence for primary visual cortex plasticity. Proceedings of the National Academy of Sciences, 88, 4966-4970.

KARNI, A., \& SAGI, D. (1993). The time course of learning a visual skill. Nature, 365, 250-252.
Kastner, S., Nothdurft, H. C., \& Pigarev, I. N. (1997). Neuronal correlates of pop-out in cat striate cortex. Vision Research, 37, 371-376.

Kumar, T., \& Glaser, D. A. (1993). Initial performance, learning and observer variability for hyperacuity tasks. Vision Research, 33, 2287-2300.

Lobley, K., \& WALSH, V. (1998). Perceptual learning in visual conjunction search. Perception, 27, 1245-1255.

Malik, J., \& Perona, P. (1990). Preattentive texture discrimination with early vision mechanisms. Journal of the Optical Society of America A, 7, 923-932.

MaYer, M. J. (1983). Practice improves adults' sensitivity to diagonals. Vision Research, 23, 547-550.

McKee, S. P., \& Westheimer, G. (1978). Improvement in vernier acuity with practice. Perception \& Psychophysics, 24, 258-262.

Nothdu RFT, H. C. (1992). Feature analysis and the role of similarity in preattentive vision. Perception \& Psychophysics, 52, 355-375.

Nothdurft, H. C. (1997). Different approaches to the coding of visual segmentation. In I. Harris \& M. Kjenkinus (Eds.), Computationaland psychophysical mechanisms of visual coding (pp. 355-375). New York: Cambridge University Press.

Rubenstein, B. S., \& SAGI, D. (1990). Spatial variability as a limiting factor in texture-discrimination tasks: Implications for performance asymmetries. Journal of the Optical Society of America A, 7, 1632-1643.

SAARINEN, J., \& LEVI, D. M. (1995). Perceptual learning in vernier acuity: What is learned? Vision Research, 35, 519-527.

SAGI, D., \& JulesZ, B. (1984). Detection versus discrimination of visual orientation. Perception, 13, 619-628.

SAGI, D., \& JulesZ, B. (1987). Short-range limitation on detection of feature differences. Spatial Vision, 2, 39-49.

Shiu, L.-P., \& PASHLER, H. (1992). Improvement in line orientation discrimination is retinally local but dependent on cognitive set. Perception \& Psychophysics, 52, 582-588.

Sireteanu, R., \& Rettenbach, R. (1995). Perceptual learning in visual search: Fast, enduring, but non-specific. Vision Research, 35, $2037-$ 2043.

Tanaka, K., Saito, H., Fukada, Y., \& Moriya, M. (1991). Coding visual images of objects in the inferotemporal cortex of macaque monkey. Journal of Neurophysiology, 66, 170-189.

Tootel, R. B. H., Silverman, M. S., \& De Valois, R. L. (1991). Spatial frequency columns in primary visual cortex. Science, 214, 813-815.

Treisman, A. M., \& Gelade, G. (1980). A feature-integration theory of attention. Cognitive Psychology, 12, 97-136.

Treisman, A. [M.], Vieira, A., \& Hayes, A. (1992). Automaticity and preattentive processing. American Journal of Psychology, 105, 342-362.

Vogels, R., \& Orban, G. A. (1985). The effect of practice on the oblique effect in line orientation judgements. Vision Research, 25, 1679-1687.

Walsh, V., Ashbridge, E., \& Cowey, A. (1998). Cortical plasticity in perceptual learning demonstrated by transcortical magnetic stimulation. Neuropsychologia, 34, 363-367.

Wolfe, J. M., Yu, K. P., Stewart, M. I., Shorter, A. D., FriedmanHILl, S. R. \& CAVE, K. R. (1990). Limitations on the parallel guidance of visual search, color $\times$ color and orientation $\times$ orientation. Journal of Experimental Psychology: Human Perception \& Performance, 16, 879-892.

\section{NOTES}

1. Note that it would be impossible to calculate any statistic based on intersubject variability (such as ANOVA), since $T$ values are intrinsically group values - that is, values obtained by comparing mean search time differences of different groups of subjects.

2. Percentage values of $T$ were used to calculate the chi-square.

(Manuscript received April 15, 2002; revision accepted for publication April 8, 2003.) 